\title{
A Minimal Poset Resolution of Stable Ideals
}

\author{
Timothy B. P. Clark
}

\begin{abstract}
We give a brief survey of the various topological and combinatorial techniques which have been used to construct the minimal free resolution of a stable monomial ideal in a polynomial ring over a field. The new results appearing in this paper describe a connection between certain topological and combinatorial methods for the description of said minimal resolutions. In particular, we construct a minimal poset resolution of an arbitrary stable monomial ideal by using a poset of Eliahou-Kervaire admissible symbols associated to a stable ideal. The structure of the poset under consideration is quite rich and in related analysis, we exhibit a regular CW complex which supports this resolution.
\end{abstract}

Keywords. Monomial Ideal, Minimal Poset Resolution, Regular CW Complex, CW Poset, Shellability.

2010 Mathematics Subject Classification. Primary: 13D02; Secondary: $05 E 40$.

\section{Introduction}

Let $R=\mathbb{k}\left[x_{1}, \ldots, x_{d}\right]$, where $\mathbb{k}$ is a field and $R$ is considered with its standard $\mathbb{Z}^{d}$ grading (multigrading). For a monomial ideal $N$ of $R$, the minimal free resolution of the module $R / N$ is a well-studied invariant whose non-recursive construction using only the field $\mathbb{k}$ and the unique monomial generators of the ideal is an open problem.

Precisely, a minimal free resolution is an exact sequence of multigraded $R$-modules connected by multigraded morphisms which encodes the minimal relations between generators of the syzygy modules of $R / N$. We denote a minimal free resolution of $R / N$ as

$$
\mathcal{F}: \cdots \longrightarrow \bigoplus_{\alpha \in \mathbb{Z}^{n}} F_{i, \alpha} \stackrel{\partial_{i}}{\longrightarrow} \bigoplus_{\alpha \in \mathbb{Z}^{n}} F_{i-1, \alpha} \longrightarrow \cdots \longrightarrow \bigoplus_{\alpha \in \mathbb{Z}^{n}} F_{1, \alpha} \stackrel{\partial_{1}}{\longrightarrow} \bigoplus_{\alpha \in \mathbb{Z}^{n}} F_{0, \alpha}
$$

where the free module $F_{i, \alpha}=R(-\alpha)^{\beta_{i, \alpha}}$ is of rank $\beta_{i, \alpha}$, the map $\partial_{i}$ is degree preserving for all $i$ and $\operatorname{Coker}\left(\partial_{1}\right) \cong R / N$.

Structure theorems for the minimal free resolution of several classes of monomial ideals have been developed in the last 20 years, although no technique has proven to be general enough to describe the minimal resolution of an arbitrary monomial ideal. Many of the approaches appearing in the literature associate to an ideal a topological or combinatorial object whose structure is shown to mirror the algebraic structure of a (minimal) resolution. Computationally speaking, a minimal free resolution of $R / N$ 
may be constructed recursively by hand or using a computer algebra system such as Macaulay2 [16].

The earliest study of resolutions of monomial ideals was described in the thesis [23] of Diana Taylor, a student of Kaplansky. For a monomial ideal with minimal generating set $\left\{m_{1}, \ldots, m_{r}\right\}$, Taylor's resolution consists of a free module of rank $\left(\begin{array}{c}r \\ k\end{array}\right)$ appearing in homological degree $k$ whose basis elements are in one-to-one correspondence with the cardinality $k$ subsets $I=\left\{i_{1}, \cdots, i_{k}\right\} \subseteq\{1, \ldots, r\}$ and have multidegree matching the monomial $m_{I}=\operatorname{lcm}\left(m_{i}: i \in I\right)$. The differential in Taylor's resolution takes the unique basis element $e_{I}$ to

$$
\sum_{j=1}^{r}(-1)^{j+1} \frac{m_{I}}{m_{I \backslash\left\{i_{j}\right\}}} \cdot e_{I \backslash\left\{i_{j}\right\}} .
$$

If one changes perspective of the Taylor resolution only slightly, the underlying vector space structure is easily recognized as the simplicial chain complex of an $r$ dimensional simplex. This re-interpretation of the Taylor resolution as an object from algebraic topology serves as an organizing example for the use of topological techniques which link the incidence structure of a regular $\mathrm{CW}$ complex with the syzygy structure of a monomial ideal.

Bayer and Sturmfels in [3] develop a program for this approach by first establishing a $\mathbb{Z}^{n}$ grading on a regular CW complex $X$ whose $r$ vertices are each associated with a generator of $N$. Indeed, for $e$, a nonempty cell of $X$, identify $e$ with the set of vertices it contains and label $e$ with the monomial $m_{e}:=\operatorname{lcm}\left\{m_{j}: j \in e\right\}$. Algebraically, the vertices contained in the cell are viewed as a finite subset of the minimal generating set of $N$.

A complex of multigraded $R$-modules, $\mathscr{F}_{N}$, is said to be a cellular resolution of $R / N$ if the following three properties are satisfied: for all $i \geq 0$ the free module $\left(\mathcal{F}_{N}\right)_{i}$ has as its basis the $i-1$ dimensional cells of $X$, a basis element $e \in\left(\mathscr{F}_{N}\right)_{i}$ has multidegree equal to that of the monomial $m_{e}$ and the differential $\partial$ of $\mathscr{F}_{N}$ acts on $e \in\left(\widetilde{F}_{N}\right)_{i}$ as

$$
\partial(e)=\sum_{\substack{e^{\prime} \subset e \subset X \\ \operatorname{dim}(e)=\operatorname{dim}\left(e^{\prime}\right)+1}} c_{e, e^{\prime}} \cdot \frac{m_{e}}{m_{e^{\prime}}} \cdot e^{\prime}
$$

where $c_{e, e^{\prime}}$ is the coefficient of the cell $e^{\prime}$ in the differential of $e$ in the cellular chain complex of $X$. Bayer and Sturmfels further show that the complex $\mathscr{F}_{N}$ is a free resolution of $N$ if and only if the subcomplex of $X$ on the vertices whose monomial labels divide $m$ is empty or acyclic over $\mathbb{k}$ for all $m \in R$.

Although this general approach is elegant, the task of determining an appropriate cell complex that supports a minimal free resolution for a monomial ideal is difficult. Moreover, Velasco [24] has constructed a class of monomial ideals whose minimal free resolution cannot be supported on any CW complex. In what follows, we therefore restrict attention to the so-called stable ideals, whose minimal resolution was first constructed explicitly by Eliahou and Kervaire [15] using combinatorial methods. Re- 
cently, these ideals were shown to have a minimal cellular resolution separately by Batzies and Welker [1] and Mermin [19]. The connections between the combinatorial technique of Eliahou and Kervaire and these two recent topological approaches comprise the original results appearing in this paper.

Eliahou and Kervaire [15] call a monomial ideal $N$ stable if for every monomial $m \in N$, the monomial $m \cdot x_{i} / x_{r} \in N$ for each $1 \leq i<r$, where $r=\max \{k$ : $x_{k}$ divides $\left.m\right\}$. They provide a construction of the minimal free resolution of a stable monomial ideal by identifying basis elements of the free modules (called admissible symbols) and describing how the maps within the resolution act on these symbols. The class of stable ideals has been extensively studied, and several of its subclasses have been shown to have relevant applications, submit to novel analytical techniques, or both.

We recall two refinements of the definition of stability. An ideal is said to be strongly stable if whenever $i<j$ and $m$ is a monomial such that $m x_{j} \in N$, it follows that $m x_{i} \in N$. Clearly, the stable ideals contain the strongly stable ideals as a subclass. When the characteristic of $\mathbb{k}$ is zero, strongly stable ideals are referred to as Borel ideals. The class of Borel ideals have been given much attention due to their importance in Gröbner Basis Theory [13].

Turning to the topological methods which have been used to describe the minimal free resolution of a stable ideal, we focus on the most general constructions appearing in the literature.

Batzies and Welker in [1] develop an application of discrete Morse theory to the reduced cellular chain complex of a $\mathbb{Z}^{d}$ graded regular $\mathrm{CW}$ complex. This technique reduces the length of and number of free modules appearing in a nonminimal cellular resolution by collapsing certain cells of the given CW complex. The (not necessarily regular) CW complex which results from this collapsing procedure is homotopy equivalent to the original regular CW complex but has fewer cells. For the class of stable monomial ideals, this approach results in the reduction of the Taylor resolution of a stable monomial ideal to a minimal cellular resolution which matches the construction of Lyubeznik [18].

Mermin in [19] studies the minimal free resolution of an arbitrary stable ideal by defining a regular CW complex whose cells are built using the variable exchange property which characterizes a stable ideal. This regular CW complex is shown to support the original minimal resolution of Eliahou and Kervaire. Furthermore, the regular CW complex construction of Mermin seems to match that of Batzies and Welker, although the precise connections between these methods have not been studied.

In addition to these general techniques, topological approaches for constructing a minimal resolution of ideals in certain subclasses of the stable ideals are also present in the literature.

Sinefakopoulos in [22] defines a Borel principal ideal as the smallest Borel-fixed monomial ideal having a fixed monomial $m$ in its generating set. The inductive construction which he describes results in a shellable, polyhedral cell complex that sup- 
ports the minimal free resolution of an arbitrary ideal from this subclass. The cellular incidence structure of this polyhedral complex is significantly different from the $\mathrm{CW}$ complexes appearing in [1] and [19].

In [17] Horwitz constructs the minimal free resolution of a Borel ideal which is generated by squarefree quadratic monomials. By reinterpreting said Borel ideal as an edge ideal, the algebraic analogues of certain graph-theoretic techniques prove to be useful in the construction of a minimal resolution. This resolution in fact has a regular cellular structure and has differential maps which coincide with those of the Eliahou-Kervaire resolution.

Using techniques which they first developed in [11], Corso and Nagel [12] recover the result of Horwitz and provide a more explicit construction for the minimal cellular resolution of an arbitrary strongly stable ideal generated in degree two. Their construction associates a strongly stable ideal to a Ferrers tableau which in turn gives rise to an associated polyhedral cell complex. This cell complex is shown to support the minimal free resolution of the strongly stable ideal in question. This technique is generalized to the class of squarefree strongly stable ideals generated in a fixed degree further by Nagel and Reiner in [21].

In this paper, we begin with a combinatorial perspective of stable ideals, whereby we construct a minimal poset resolution of an arbitrary stable ideal $N$. Precisely, we define a poset $\left(P_{N},<\right)$ on the admissible symbols of Eliahou and Kervaire by taking advantage of a decomposition property unique to the monomials contained in stable ideals.

In Section 2, we review the fundamentals of poset resolutions and define the poset of admissible symbols $P_{N}$. In our first main result, Theorem 2.4, we recover the EliahouKervaire resolution of a stable ideal as a poset resolution. The value of this technique lies in the structural fact that the maps in the resolution act on the basis elements of the free modules in a way that mirrors the covering relations in $P_{N}$. Considering the lattice-linear ideals of [9], poset resolutions provide a common perspective from which to view the minimal resolutions of three large and well-studied classes of monomial ideals; stable ideals, Scarf ideals [2] and ideals having a linear resolution [14].

An advantage of the method described herein is that for a fixed stable ideal, the combinatorial information contained in the poset of admissible symbols can be transformed into the topological incidence structure of a regular $\mathrm{CW}$ complex. Specifically, the poset of admissible symbols $P_{N}$ is a $C W$ poset in the sense of Björner [5], so that it is the face poset of a regular CW complex $X_{N}$.

In our second main result, Theorem 6.4, we show that $X_{N}$ supports a minimal cellular resolution of the stable ideal $N$. By using this combinatorial connection, we recover a minimal cellular resolution of $N$ in a manner distinct from two of the previously described methods. Indeed, the cell complex that comes as a consequence of Björner's correspondence coincides with the one produced in the work of Mermin [19] and appears to match the cell complex of Batzies and Welker [1]. The details of these connections are the subject of future research. 


\section{Poset Resolutions and Stable Ideals}

Let $(P,<)$ be a finite poset with set of atoms $A$ and write $\beta \lessdot \alpha$ if $\beta<\alpha$ and there is no $\gamma \in P$ such that $\beta<\gamma<\alpha$. We say that $\beta$ is covered by $\alpha$ in this situation. For $\alpha \in P$, write the order complex of the associated open interval as $\Delta_{\alpha}=\Delta(\hat{0}, \alpha)$. In [9], the collection of simplicial complexes

$$
\left\{\Delta_{\alpha}: \alpha \in P\right\}
$$

is used to construct a sequence of vector spaces and vector space maps

$$
\mathcal{D}(P): \cdots \longrightarrow \mathcal{D}_{i} \stackrel{\varphi_{i}}{\longrightarrow} \mathcal{D}_{i-1} \longrightarrow \cdots \longrightarrow \mathcal{D}_{1} \stackrel{\varphi_{1}}{\longrightarrow} \mathcal{D}_{0} .
$$

For $i \geq 1$, the vector space $\mathscr{D}_{i}$ is defined as

$$
\mathscr{D}_{i}=\bigoplus_{\alpha \in P \backslash\{\hat{0}\}} \mathscr{D}_{i, \alpha},
$$

where $\mathscr{D}_{i, \alpha}=\widetilde{H}_{i-2}\left(\Delta_{\alpha}, \mathbb{k}\right)$. In particular, the vector space $\mathscr{D}_{1}$ has its basis indexed by the set of atoms $A$ in $P$. For notational simplicity, when $\lambda \lessdot \alpha$ let $\mathbf{D}_{\lambda}=\Delta(\hat{0}, \lambda]$ and

$$
\Delta_{\alpha, \lambda}=\mathbf{D}_{\lambda} \cap\left(\bigcup_{\substack{\beta<\alpha \\ \lambda \neq \beta}} \mathbf{D}_{\beta}\right)
$$

When $i \geq 2$, the maps $\varphi_{i}$ are defined using the maps in the Mayer-Vietoris long exact sequence in reduced homology associated to the short exact sequence of reduced simplicial chain complexes

$$
0 \rightarrow \widetilde{\mathscr{e}}_{i}\left(\Delta_{\alpha, \lambda}\right) \rightarrow \widetilde{\mathscr{e}}_{i}\left(\mathbf{D}_{\lambda}\right) \oplus \widetilde{\mathscr{e}}_{n}\left(\bigcup_{\substack{\beta<\alpha \\ \lambda \neq \beta}} \mathbf{D}_{\beta}\right) \rightarrow \widetilde{\mathscr{e}}_{i}\left(\Delta_{\alpha}\right) \rightarrow 0
$$

where the triple under consideration is

$$
\left(\mathbf{D}_{\lambda}, \bigcup_{\substack{\beta<\alpha \\ \lambda \neq \beta}} \mathbf{D}_{\beta}, \Delta_{\alpha}\right)
$$

For $i \geq 2$ we write $\iota: \widetilde{H}_{i-3}\left(\Delta_{\alpha, \lambda}, \mathbb{k}\right) \rightarrow \widetilde{H}_{i-3}\left(\Delta_{\lambda}, \mathbb{k}\right)$ for the map induced in homology by the inclusion map and

$$
\delta_{i-2}^{\alpha, \lambda}: \widetilde{H}_{i-2}\left(\Delta_{\alpha}, \mathbb{k}\right) \rightarrow \widetilde{H}_{i-3}\left(\Delta_{\alpha, \lambda}, \mathbb{k}\right)
$$

for the connecting homomorphism from the Mayer-Vietoris sequence in homology of (2.1). Set

$$
\varphi_{i}^{\alpha, \lambda}: \mathscr{D}_{i, \alpha} \rightarrow \mathscr{D}_{i-1, \lambda}
$$


as the composition $\varphi_{i}^{\alpha, \lambda}=\iota \circ \delta_{i-2}^{\alpha, \lambda}$. The map $\varphi_{i}: \mathscr{D}_{i} \rightarrow \mathscr{D}_{i-1}$ is then defined componentwise by

$$
\left.\varphi_{i}\right|_{D_{i, \alpha}}=\sum_{\lambda \ll \alpha} \varphi_{i}^{\alpha, \lambda}
$$

For $i=0$, we define a one-dimensional vector space as $\mathscr{D}_{0}=\widetilde{H}_{-1}(\{\varnothing\}, \mathbb{k})$ and define $\varphi_{1}: \mathscr{D}_{1} \rightarrow \mathscr{D}_{0}$ componentwise as $\left.\varphi_{1}\right|_{\mathscr{D}_{1, \alpha}}=\operatorname{id}_{\widetilde{H}_{-1}(\{\varnothing\}, \mathbb{k})}$.

We now describe the process by which the sequence of vector spaces $\mathscr{D}(P)$ is transformed into a sequence of multigraded modules. For a monomial $m=x_{1}^{\mathbf{a}_{1}} \cdots x_{d}^{\mathbf{a}_{d}} \in R$ we write $\operatorname{mdeg}(m)=\left(\mathbf{a}_{1}, \ldots, \mathbf{a}_{d}\right)$ and $\operatorname{deg}_{x_{\ell}}(m)=\mathbf{a}_{\ell}$ for $1 \leq \ell \leq d$. Assuming the existence of a map of partially ordered sets $\eta: P \longrightarrow \mathbb{N}^{n}$, the sequence of vector spaces $\mathscr{D}(P)$ is homogenized to produce

$$
\mathscr{F}(\eta): \cdots \longrightarrow \mathscr{F}_{t} \stackrel{\partial_{t}}{\longrightarrow} \mathscr{F}_{t-1} \longrightarrow \cdots \longrightarrow \mathscr{F}_{1} \stackrel{\partial_{1}}{\longrightarrow} \mathscr{F}_{0},
$$

a sequence of free multigraded $R$-modules and multigraded $R$-module homomorphisms.

For $i \geq 1$, we set

$$
\mathcal{F}_{i}=\bigoplus_{\hat{0} \neq \lambda \in P} \mathcal{F}_{i, \lambda}=\bigoplus_{\hat{0} \neq \lambda \in P} R \otimes_{\mathbb{k}} D_{i, \lambda}
$$

where the grading is defined as $\operatorname{mdeg}\left(x^{\mathbf{a}} \otimes v\right)=\mathbf{a}+\eta(\lambda)$ for each $v \in \mathscr{D}_{i, \lambda}$.

The differential $\partial_{i}: \mathscr{F}_{i} \longrightarrow \mathscr{F}_{i-1}$ in this sequence of multigraded modules is defined as

$$
\left.\partial_{i}\right|_{\mathscr{F}_{i, \alpha}}=\sum_{\lambda \ll \alpha} \partial_{i}^{\alpha, \lambda}
$$

where $\partial_{i}^{\alpha, \lambda}: \mathscr{F}_{i, \alpha} \longrightarrow \mathcal{F}_{i-1, \lambda}$ takes the form $\partial_{i}^{\alpha, \lambda}=x^{\eta(\alpha)-\eta(\lambda)} \otimes \varphi_{i}^{\alpha, \lambda}$ for $\lambda \lessdot \alpha$.

We set $F_{0}=R \otimes_{\mathbb{k}} D_{0}$ and multigrade the result with $\operatorname{mdeg}\left(x^{\mathbf{a}} \otimes v\right)=\mathbf{a}$ for each $v \in \mathscr{D}_{0}$. The differential $\partial_{1}^{\alpha, \lambda}: \mathscr{F}_{1, \alpha} \longrightarrow \mathcal{F}_{0, \lambda}$ is defined componentwise as

$$
\left.\partial_{1}\right|_{F_{1, \lambda}}=\left.x^{\eta(\lambda)} \otimes \varphi_{1}\right|_{D_{1, \lambda}} .
$$

The sequence $\mathcal{F}(\eta)$ approximates a free resolution of the multigraded module $R / M$ where $M$ is the ideal in $R$ generated by the monomials

$$
\left\{x^{\eta(a)}: a \in A\right\}
$$

whose multidegrees are given by the images of the atoms of $P$.

Definition 2.1 ([9]). If $\mathcal{F}(\eta)$ is an acyclic complex of multigraded modules, then we say that it is a poset resolution of the ideal $M$. 
Throughout the remainder of the paper, $N$ will denote a stable monomial ideal in $R$ and we write $G(N)$ as the unique minimal generating set of $N$. For a monomial $m \in R$ set

$$
\max (m)=\max \left\{k \mid x_{k} \text { divides } m\right\}
$$

and

$$
\min (m)=\min \left\{k \mid x_{k} \text { divides } m\right\}
$$

To describe further the class of stable ideals, let $[d-1]=\{1, \ldots, d-1\}$, for $I \subseteq[d-1]$ let $\max (I)=\max \{i \mid i \in I\}$ and write $x_{I}=\prod_{i \in I} x_{i}$.

In Lemma 1.2 of [15], Eliahou and Kervaire prove that a monomial ideal $N$ is stable if and only if for each monomial $m \in N$ there exists a unique $n \in G(N)$ with the property that $m=n \cdot y$ and $\max (n) \leq \min (y)$. We adopt the language and notation introduced in the paper of Eliahou and Kervaire, and refer to $n$ as the unique decomposition of the monomial $m$. Following their convention, we encode this property in a decomposition map $\mathbf{g}: M(N) \longrightarrow G(N)$ where $M(N)$ is the collection of monomials of $N$ and $\mathbf{g}(m)=n$.

Definition 2.2 ([15]). An admissible symbol is an ordered pair $(I, m)$ which satisfies $\max (I)<\max (m)$, where $m \in G(N)$ and $I \subseteq[d-1]$.

Definition 2.3. The poset of admissible symbols is the set $P_{N}$ of all admissible symbols associated to $N$, along with the symbol $\hat{0}=(\varnothing, 1)$ which is defined to be the minimum element of $P_{N}$. The partial ordering on $P_{N}$ is

$$
\begin{aligned}
(J, n) \leq(I, m) \Longleftrightarrow \quad & J \subseteq I \text { and there exists } \\
& C \subseteq I \backslash J \text { so that } n=\mathbf{g}\left(x_{C} m\right)
\end{aligned}
$$

when both symbols are admissible.

In the case when $(J, n)<(I, m)$ and $I=J \cup\{\ell\}$ for some $\ell$, then we write $(J, n) \lessdot(I, m)$ to describe the covering that occurs in $P_{N}$. As constructed, we have $\hat{0} \lessdot(\varnothing, m)$ for every $m \in G(N)$. We are now in a position to state our first main result.

Theorem 2.4. Suppose that $N$ is a stable monomial ideal with poset of admissible symbols $P_{N}$ and define the map $\eta: P_{N} \longrightarrow \mathbb{N}^{n}$ so that $(I, m) \mapsto \operatorname{mdeg}\left(x_{I} m\right)$. Then the complex $\mathcal{F}(\eta)$ is a minimal poset resolution of $R / N$.

In order to prove Theorem 2.4, we first describe the combinatorial structure of $P_{N}$ and then exhibit the connection between the complex $\mathcal{F}(\eta)$ and the minimal free resolution of the stable ideal $N$ constructed by Eliahou and Kervaire in [15]. 


\section{The Shellability of $\boldsymbol{P}_{N}$}

We begin this section by recalling some general facts regarding the shellability of partially ordered sets. Recall that a poset $P$ is called shellable if the facets of its order complex $\Delta(P)$ can be arranged in a linear order $F_{1}, F_{2}, \ldots, F_{t}$ in such a way that the subcomplex

$$
\left(\bigcup_{i=1}^{k-1} F_{i}\right) \cap F_{k}
$$

is a nonempty union of maximal proper faces of $F_{k}$ for $k=2, \ldots, t$. Such an ordering of facets is called a shelling.

Definition 3.1. Let $\mathcal{E}(P)$ denote the collection of edges in the Hasse diagram of a poset $P$. An edge labeling of $P$ is a map $\lambda: \mathscr{E}(P) \longrightarrow \Lambda$ where $\Lambda$ is some poset.

For $\sigma=a_{1} \lessdot \cdots \lessdot a_{k}$, a maximal chain of $P$, the edge label of $\sigma$ is the sequence of labels $\lambda(\sigma)=\left(\lambda\left(a_{1} \lessdot a_{2}\right), \ldots, \lambda\left(a_{k-1} \lessdot a_{k}\right)\right)$.

Definition 3.2. An edge labeling $\lambda$ is called an EL-labeling (edge lexicographical labeling) if for every interval $[x, y]$ in $P$,

(i) there is a unique maximal chain $\sigma$ in $[x, y]$, such that the labels of $\sigma$ form an increasing sequence in $\Lambda$. We call $\sigma$ the unique increasing maximal chain in $[x, y]$.

(ii) $\lambda(\sigma)<\lambda\left(\sigma^{\prime}\right)$ under the lexicographic partial ordering in $\Lambda$ for all other maximal chains $\sigma^{\prime}$ in $[x, y]$.

A graded poset that admits an EL-labeling is said to be EL-shellable (edge lexicographically shellable).

We further recall the following fundamental result of Björner and Wachs.

Theorem 3.3 ([7]). EL-shellable posets are shellable.

We now define an edge labeling of the poset of admissible symbols $P_{N}$.

Definition 3.4. Let $\lambda: P_{N} \rightarrow \mathbb{Z}$ take the form

$$
\lambda((J, n) \lessdot(I, m))= \begin{cases}0 & \text { if } n=1 \\ -\ell & \text { if } n=m \\ \ell & \text { if } n \neq m,\end{cases}
$$

where $\{\ell\}=I \backslash J$.

Example 3.5. The labeled Hasse diagram for the poset of admissible symbols, $P_{N}$, of the stable ideal $N=\langle a, b, c\rangle^{2}=\left\langle a^{2}, a b, a c, b^{2}, b c, c^{2}\right\rangle$ is 


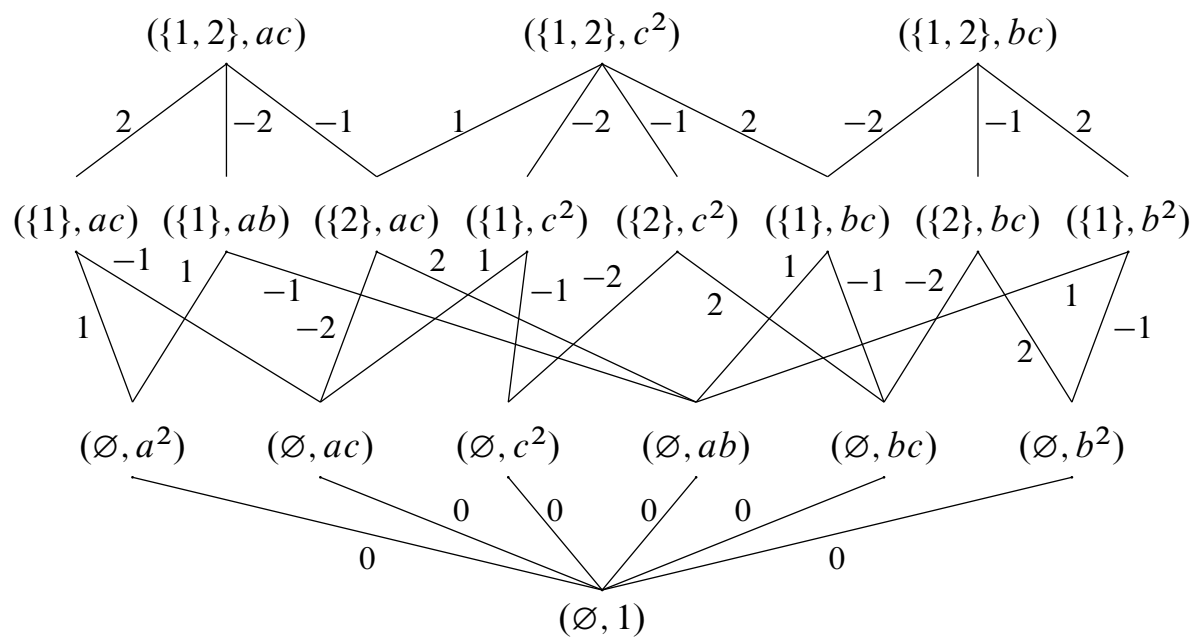

Recall that given a poset $P$, the dual poset $P^{*}$ has an underlying set identical to that of $P$, with $x<y$ in $P^{*}$ if and only if $y<x$ in $P$. Further, an edge labeling of a poset $P$ may also be viewed as an edge labeling of its dual poset and $P$ is said to be dual shellable if $P^{*}$ is a shellable poset.

Theorem 3.6. The poset $P_{N}$ is dual EL-shellable with $\lambda$ defined as above.

Before turning to the proof of Theorem 3.6, we discuss some properties of the decomposition map $\mathbf{g}$ and the edge labeling $\lambda$.

\section{Remarks 3.1.}

(i) [15, Lemma 1.3] For any monomial $w$ and any monomial $m \in N$, we have $\mathbf{g}(w \mathbf{g}(m))=\mathbf{g}(w m)$ and $\max (\mathbf{g}(w m)) \leq \max (\mathbf{g}(m))$. We refer to the first property as the associativity of $\mathbf{g}$.

(ii) Suppose that $[(I, m),(J, n)]$ is a closed interval in the dual poset $P_{N}^{*}$. Given a sequence of labels

$$
\left(l_{1}, \ldots, l_{k}\right)
$$

there is at most one maximal chain $\sigma$ in the closed interval such that

$$
\lambda(\sigma)=\left(l_{1}, \ldots, l_{k}\right) .
$$

When it exists, this chain must be equal to

$$
(I, m) \lessdot\left(I \backslash\left\{\ell_{1}\right\}, n_{1}\right) \lessdot \cdots \lessdot\left(I \backslash\left\{\ell_{1}, \ldots, \ell_{k-1}\right\}, n_{k-1}\right) \lessdot(J, n)
$$

where $\ell_{i}=\left|l_{i}\right|$, the set $I \backslash J=\left\{\ell_{1}, \ldots, \ell_{k}\right\}$ and

$$
n_{i}= \begin{cases}\mathbf{g}\left(x_{\ell_{i}} n_{i-1}\right) & \text { if } l_{i}>0 \\ n_{i-1} & \text { if } l_{i}<0\end{cases}
$$

for $1 \leq i \leq k$ with $n_{0}=m$ and $n_{k}=n$. 
Suppose that $(J, n)<(I, m)$ is a pair of comparable admissible symbols. Then $n=$ $\mathbf{g}\left(x_{C^{\prime}} m\right)$ for some $C^{\prime} \subseteq I \backslash J$. Let $C=\left\{c \in C^{\prime} \mid c \leq \max (n)\right\}$. Then by associativity and [15, Lemma 1.2] we have $n=\mathbf{g}\left(x_{C^{\prime}} m\right)=\mathbf{g}\left(x_{C^{\prime} \backslash C} \mathbf{g}\left(x_{C} m\right)\right)=\mathbf{g}\left(x_{C} m\right)$. In this way, any representation of $n=\mathbf{g}\left(x_{C^{\prime}} m\right)$ may be reduced to $n=\mathbf{g}\left(x_{C} m\right)$ under the conditions above.

Notation 3.2. Implicit in all subsequent arguments is the convention that a representation $n=\mathbf{g}\left(x_{C} m\right)$ is written in reduced form.

Lemma 3.7. For a reduced representation of $n=\mathbf{g}\left(x_{C} m\right)$ the set $C$ is the unique subset of minimum cardinality among all $C^{\prime} \subseteq I \backslash J$ for which $n=\mathbf{g}\left(x_{C^{\prime}} m\right)$.

Proof. Suppose that $C$ is not the subset of $I \backslash J$ with smallest cardinality, namely that there exists $D \subseteq I \backslash J$ with $|D|<|C|$ and $n=\mathbf{g}\left(x_{D} m\right)$. By definition, $x_{C} \cdot m=$ $n \cdot y$ and $x_{D} \cdot m=n \cdot u$ where $\max (n) \leq \min (y)$ and $\max (n) \leq \min (u)$. The assumption of $|D|<|C|$ implies that there exists $c \in C$ such that $c \notin D$. Rearranging and combining the two equations above, we arrive at the equality $x_{C} \cdot u=x_{D} \cdot y$. This equality allows us to conclude that $x_{c}$ divides $y$ since it cannot divide $x_{D}$. By definition, $\max (n) \leq \min (y)$ and therefore $\max (n) \leq c$. Further, since we assumed that $n=\mathbf{g}\left(x_{C} m\right)$ possessed the property that $c \leq \max (n)$ we have $c \leq \max (n) \leq c$ so that $\max (n)=c$. This equality also has implications for $x_{D}$ and $u$, namely that $c \leq \min (u)$ and $\max (D) \leq \max (n)=c$ so that $\max (D)<c$ since $c \notin D$. However, $c<\max (D) \leq \max (n)=c$ is a contradiction, and our original supposition that such a $D$ exists is false. If $C$ and $D$ are distinct subsets of $I \backslash J$ with $|C|=|D|$ and $n=\mathbf{g}\left(x_{C} m\right)=\mathbf{g}\left(x_{D} m\right)$ then there is a $c \in C$ and $d \in D$ for which $c \notin D$ and $d \notin C$. As before, we use the equality $x_{C} \cdot u=x_{D} \cdot y$ and now conclude that $x_{c}$ divides $y$ and $x_{d}$ divides $u$. Therefore, $c \leq \max (C) \leq \max (n) \leq \min (y) \leq c$ and similarly $d \leq \max (D) \leq \max (n) \leq \min (u) \leq d$ so that $\max (n)=c=d$,

Proof of Theorem 3.6. To prove the dual EL-Shellability of $P_{N}$, recall that for the poset of admissible symbols $P_{N}$, we have comparability in the dual poset given by $(I, m)<(J, n) \in P_{N}^{*}$ if and only if $(J, n)<(I, m) \in P_{N}$. We proceed with the proof by considering the various types of closed intervals that appear in the dual poset $P_{N}^{*}$.

Case 1: Consider the closed interval $[(I, m), \hat{0}]$. Write $I=\left\{d_{1}, \ldots, d_{t}\right\}$ so that $d_{j}<d_{j+1}$, for every $j=1, \ldots, t$. The maximal chain

$$
\sigma=(I, m) \lessdot\left(I \backslash\left\{d_{t}\right\}, m\right) \lessdot\left(I \backslash\left\{d_{t}, d_{t-1}\right\}, m\right) \lessdot \cdots \lessdot(\varnothing, m) \lessdot \hat{0}
$$

has the increasing label

$$
\left(-d_{t},-d_{t-1}, \ldots,-d_{1}, 0\right) .
$$

Consider a maximal chain $\tau \in[(I, m), \hat{0}]$ where $\tau \neq \sigma$. If each label in the sequence $\lambda(\tau)$ (except the label of coverings of the form $(\varnothing, n) \lessdot \hat{0}$ ) is negative, then the 
sequence $\lambda(\tau)$ cannot be increasing, for it must be a permutation of the sequence $\lambda(\sigma)$ where the rightmost label 0 is fixed. If any label within the sequence $\lambda(\tau)$ is positive, then again $\lambda(\tau)$ cannot be increasing since every maximal chain contains the labeled subchain

$$
(\varnothing, n) \stackrel{0}{\leftarrow} \hat{0}
$$

for every $(I, m)<(\varnothing, n)$. Therefore, $\sigma$ is the unique rising chain in the interval $[(I, m), \hat{0}]$. Further, $\lambda(\sigma)$ is lexicographically first among all chains in $[(I, m), \hat{0}]$ since $-d_{t}<\cdots<-d_{1}<0$.

Case 2: Consider the closed interval $[(I, m),(J, n)]$ of $P_{N}^{*}$ where $(J, n) \neq \hat{0}$ and $n=m$. Again write $I \backslash J=\left\{d_{1}, \ldots, d_{t}\right\}$ such that $d_{1}<\cdots<d_{t}$. Every maximal chain $\sigma$ in $[(I, m),(J, m)]$ has a label of the form

$$
\left(-d_{\rho(t)}, \ldots,-d_{\rho(1)}\right)
$$

where $\rho \in \Sigma_{t}$ is a permutation of the set $\{1, \ldots, t\}$. Therefore, the label

$$
\left(-d_{t}, \ldots,-d_{1}\right)
$$

corresponding to the identity permutation is the unique increasing label in the interval $[(I, m),(J, m)]$ and is lexicographically first among all such labels.

Case 3: Consider the closed interval $[(I, m),(J, n)]$ of $P_{N}^{*}$ where $(J, n) \neq \hat{0}$ and $m \neq n$. By Lemma 3.7, $n=\mathbf{g}\left(x_{C} m\right)$ for a unique $C \subseteq I \backslash J$ where $\max (C) \leq$ $\max (n)$ and the set $C$ is of minimum cardinality. Writing the set $C=\left\{c_{1}, \ldots, c_{q}\right\}$ and $(I \backslash J) \backslash C=\left\{\ell_{1}, \ldots, \ell_{t}\right\}$ where $\ell_{1}<\cdots<\ell_{t}$ and $c_{1}<\cdots<c_{q}$, it follows that the sequence of edge labels $\left(-\ell_{t}, \ldots,-\ell_{1}, c_{1}, \ldots, c_{q}\right)$ is the increasing label of a maximal chain $\sigma$ in $[(I, m),(J, n)]$.

Turning to uniqueness, suppose that $\tau \neq \sigma$ is also a chain which has a rising edge label. Then

$$
\lambda(\tau)=\left(-d_{p}, \ldots,-d_{1}, s_{1}, \ldots, s_{j}\right)
$$

where

$$
\left\{s_{1}, \ldots, s_{j}\right\} \cup\left\{d_{1}, \ldots, d_{p}\right\}=\left\{c_{1}, \ldots, c_{q}\right\} \cup\left\{\ell_{1}, \ldots, \ell_{t}\right\}=I \backslash J,
$$

and $-d_{p}<\ldots<-d_{1}<0<s_{1}<\ldots<s_{j}$. Since $\tau \neq \sigma$, then $\lambda(\tau) \neq \lambda(\sigma)$ and in particular, $\left\{d_{1}, \ldots, d_{p}\right\} \neq\left\{\ell_{1}, \ldots, \ell_{t}\right\}$.

If there exists $\ell \in\left\{\ell_{1}, \ldots, \ell_{t}\right\}$ with the property that $\ell \notin\left\{d_{1}, \ldots, d_{p}\right\}$, we must have $\ell \in\left\{s_{1}, \ldots, s_{j}\right\}$ so that $\ell=s_{i}$ for some $i<j$ and the label $\lambda(\sigma)$ has the form

$$
\left(-d_{p}, \ldots,-d_{1}, s_{1}, \ldots, \ell, \ldots, s_{j}\right)
$$

By the definition of $\mathbf{g}$, we have the equalities $x_{C} \cdot m=n \cdot y$ and $x_{S} \cdot m=n \cdot u$, which may be combined and simplified to arrive at the equation $x_{C} \cdot u=x_{S} \cdot y$. The assumption that $\ell \in S$ and $\ell \notin C$ implies that $x_{\ell}$ divides $u$ so that $\max (n) \leq \ell$. 
It therefore follows that $\max (n) \leq \ell<s_{i+1}<\cdots<s_{j}$ when $\ell \neq s_{j}$ so that $\max \left(I \backslash\left\{s_{1}, \ldots, \ell\right\}\right)=s_{j}>\max (n)$, which contradicts the admissibility of the symbol $\left(I \backslash\left\{d_{1}, \ldots, d_{p}, s_{1}, \ldots, \ell\right\}, n\right)$. If $\ell=s_{j}$ then $\max (n) \leq s_{j}$ and therefore $n=\mathbf{g}\left(x_{s_{1}} \cdots x_{j} m\right)=\mathbf{g}\left(x_{s_{1}} \cdots x_{j-1} m\right)$ which implies that the symbol $(I \backslash$ $\left.\left\{s_{1}, \ldots, s_{j-1}\right\}, \mathbf{g}\left(x_{s_{1}} \cdots x_{j-1} m\right)\right)$, preceding $(J, n)$ in the chain is not admissible. If there exists $d_{g} \in\left\{d_{1}, \ldots, d_{p}\right\}$ with $d_{g} \notin\left\{\ell_{1}, \ldots, \ell_{t}\right\}$ then a similar argument again provides a contradiction to admissibility.

We now prove that $\lambda(\sigma)$ is lexicographically smallest among all chains. Aiming for a contradiction, suppose that the label $\lambda(\sigma)$ is not lexicographically smallest so that there exists a maximal dual chain $\tau$ with $\lambda(\tau)<\lambda(\sigma)$. Without loss of generality, we may assume that $\lambda(\sigma)$ and $\lambda(\tau)$ differ at their leftmost label $-c$, where $-c<-\ell_{t}$. Such a $c$ must be an element of the set $C$ since $-\ell_{t}<\cdots<-\ell_{1}$ is inherent in the structure of $\lambda(\sigma)$. By construction, $c \in C$ implies that $c \leq \max (n)$ and utilizing the equations $x_{C} \cdot m=n \cdot y$ and $x_{S} \cdot m=n \cdot u$, to produce $x_{C} \cdot u=x_{S} \cdot y$, it follows that $x_{c}$ divides $y$ and therefore $c \leq \max (n) \leq \min (y) \leq c$ so that $\max (n)=c$. This forces the element $c=c_{q}$ for otherwise, the chain $\sigma$ would contain the subchain $\left(I \backslash\left\{\ell_{1}, \ldots, \ell_{t}, c_{1}, \ldots, c\right\}, n\right)<(I \backslash J, n)$ where $\left(I \backslash\left\{\ell_{1}, \ldots, \ell_{t}, c_{1}, \ldots, c\right\}, n\right)$ is not an admissible symbol.

The desired contradiction will be obtained within an investigation of each of the three possibilities for the relationship between $\operatorname{deg}_{x_{c}}(n)$ and $\operatorname{deg}_{x_{c}}(m)$.

Suppose $\operatorname{deg}_{x_{c}}(n)>\operatorname{deg}_{x_{c}}(m)$ so that $\operatorname{deg}_{x_{c}}(n)=\operatorname{deg}_{x_{c}}(m)+1$, based upon the structure of the set $I$ and the definition of the decomposition map g. In this case, the chain $\tau$ cannot end in $(J, n)$ since $-c$, the leftmost label of $\tau$, labels the subchain $(I, m) \lessdot(I \backslash\{c\}, m)$ and the $x_{c}$ degree of every monomial appearing in the chain $\tau$ may not increase.

If $\operatorname{deg}_{x_{c}}(n)<\operatorname{deg}_{x_{c}}(m)$ then the unique decomposition $x_{C} \cdot m=n \cdot u$ implies that $x_{c}$ divides $u$, for otherwise $c \in C$ implies that $\operatorname{deg}_{x_{c}}(n)=\operatorname{deg}_{x_{c}}(m)+1$, a contradiction. The conclusion that $x_{c}$ divides $u$ allows $x_{C} \cdot m=n \cdot u$ to be simplified to $x_{C^{\prime}} \cdot m=n \cdot u^{\prime}$ where $C^{\prime}=C \backslash\{c\}$ and $u^{\prime}=u / x_{c}$. This contradicts the condition that $C$ is the set of smallest cardinality for which $\mathbf{g}\left(x_{C} m\right)=n$.

Lastly, if $\operatorname{deg}_{x_{c}}(n)=\operatorname{deg}_{x_{c}}(m)$ we turn to the chain $\sigma$, whose rightmost label is $c$. The subchain with this label is $\left(I \backslash\left\{\ell_{1}, \ldots, \ell_{t}, c_{1}, \ldots, c_{j-1}\right\}, n^{\prime}\right)<(I \backslash J, n)$ where $x_{c} \cdot n^{\prime}=n \cdot y$ where $n$ does not contain this new factor of $x_{c}$. The monomial $x_{c}$ therefore divides $y$ and we can reduce $x_{c} \cdot n^{\prime}=n \cdot y$ to $n^{\prime}=n \cdot u^{\prime}$ where $u^{\prime}=u / x_{c}$, a contradiction to $n^{\prime} \in G(N)$. This completes the proof.

With Theorem 3.6 established, we immediately have the following corollary.

Corollary 3.8. Every interval of $P_{N}$ which is of the form $[\hat{0},(I, m)]$ is finite, dual EL-shellable and therefore shellable. 


\section{The topology of $P_{N}$ and properties of $\mathscr{D}\left(P_{N}\right)$}

To establish the connection between the poset $P_{N}$ and the sequence $\mathcal{D}\left(P_{N}\right)$, we recall the definition of $C W$ poset, due to Björner [5].

Definition 4.1 ([5]). A poset $P$ is called a $C W$ poset if

(i) $P$ has a least element $\hat{0}$,

(ii) $P$ is nontrivial (has more than one element),

(iii) For all $x \in P \backslash\{\hat{0}\}$, the open interval $(\hat{0}, x)$ is homeomorphic to a sphere.

After establishing this definition, Björner describes sufficient conditions for a poset to be a $\mathrm{CW}$ poset.

Proposition 4.2 ([5, Proposition 2.2]). Suppose that $P$ is a nontrivial poset such that

(i) $P$ has a least element $\hat{0}$,

(ii) every interval $[x, y]$ of length two has cardinality four,

(iii) For every $x \in P$ the interval $[\hat{0}, x]$ is finite and shellable.

Then $P$ is a $C W$ poset.

With this proposition in hand, we now may conclude the following about the structure of $P_{N}$, the poset of admissible symbols.

Theorem 4.3. The poset of admissible symbols $P_{N}$ is a CW poset.

Proof. The poset $P_{N}$ has a least element by construction and each of its intervals $[\hat{0},(I, m)]$ is finite and shellable by Corollary 3.8. Thus, it remains to show that every closed interval in $P_{N}$ of length two has cardinality four.

Case 1: Let $(J, n)=\hat{0}$ so that the set $I$ is a singleton. It follows that the only poset elements in the open interior of the interval are $(\varnothing, m)$ and $\left(\varnothing, \mathbf{g}\left(x_{I} m\right)\right)$.

Case 2: Let $(J, n) \neq \hat{0}$ and suppose that $[(J, n),(I, m)]$ is a closed interval of length two in the poset of admissible symbols, $P_{N}$. Since the interval is of length two, the set $J$ has the form $I \backslash\left\{i_{0}, i_{1}\right\}$ for some $i_{0}<i_{1} \in I$. Further, any poset element in the interval must have either $I \backslash\left\{i_{0}\right\}$ or $I \backslash\left\{i_{1}\right\}$ as its first coordinate, for these sets are the only subsets of $I$ which contain $I \backslash\left\{i_{0}, i_{1}\right\}$.

Write $m=m^{\prime} x_{i_{2}} x_{i_{3}}$ where $\max \left(m^{\prime}\right) \leq i_{2} \leq i_{3}$. We must now consider each of the possible orderings for the elements of the (multi) set $\left\{i_{0}, i_{1}, i_{2}, i_{3}\right\}$ to ascertain the choices available for the monomial $n$. Our assumptions of the inequalities $i_{0}<i_{1}$ and $i_{2} \leq i_{3}$ together with the admissibility of the symbol $(I, m)$ imply that $i_{1} \leq \max (I)<$ $\max (m) \leq i_{3}$. Hence, determining the number of orderings amounts to producing a count of the number of orderings for elements of the set $\left\{i_{0}, i_{1}, i_{2}\right\}$, of which there are three, since $i_{0}<i_{1}$. 
Subcase 2.1: Suppose that $i_{0}<i_{1}<i_{2} \leq i_{3}$.

If $n=m$, then the poset elements which are contained in the open interior of the interval are forced to be $\left(I \backslash\left\{i_{0}\right\}, m\right)$ and $\left(I \backslash\left\{i_{1}\right\}, m\right)$.

If $n=\mathbf{g}\left(x_{i_{0}} m\right)$ and $\max \left(I \backslash\left\{i_{0}\right\}\right)<\max \left(\mathbf{g}\left(x_{i_{0}} m\right)\right)$ then the symbol $\left(I \backslash\left\{i_{0}\right\}\right.$, $\left.\mathbf{g}\left(x_{i_{0}} m\right)\right)$ is admissible, so that it is in the open interior of the interval along with the admissible symbol $\left(I \backslash\left\{i_{1}\right\}, m\right)$. The symbol $\left(I \backslash\left\{i_{0}\right\}, m\right)$ is not comparable to $\left(I \backslash\left\{i_{0}\right\}, \mathbf{g}\left(x_{i_{0}} m\right)\right)$ due to the absence of the value $i_{0}$. The symbol $\left(I \backslash\left\{i_{1}\right\}, \mathbf{g}\left(x_{i_{1}} m\right)\right)$ is also not comparable to $\left(I \backslash\left\{i_{0}\right\}, \mathbf{g}\left(x_{i_{0}} m\right)\right)$, for if it were then either $\mathbf{g}\left(x_{i_{0}} m\right)=$ $\mathbf{g}\left(x_{\varnothing} \mathbf{g}\left(x_{i_{1}} m\right)\right)=\mathbf{g}\left(x_{i_{1}} m\right)$ or $\mathbf{g}\left(x_{i_{0}} x_{i_{1}} m\right)=n=\mathbf{g}\left(x_{i_{0}} m\right)$. The first equality is impossible since Lemma 3.7 guarantees that $\left\{i_{0}\right\}$ is the unique set containing one element for which $n=\mathbf{g}\left(x_{i_{0}} m\right)$. The second equality also can not occur since Lemma 1.2 of [15] guarantees monomial equality $\mathbf{g}\left(x_{i_{0}} x_{i_{1}} m\right)=\mathbf{g}\left(x_{i_{0}} m\right)$ if and only if $\max (n) \leq \min \left(x_{i_{1}}\right)=i_{1}$, which would contradict the assumption that $\left(I \backslash\left\{i_{0}\right\}, n\right)$ is an admissible symbol.

If $n=\mathbf{g}\left(x_{i_{0}} m\right)$ and $\max \left(I \backslash\left\{i_{0}\right\}\right) \geq \max \left(\mathbf{g}\left(x_{i_{0}} m\right)\right)$ then the symbol $\left(I \backslash\left\{i_{0}\right\}\right.$, $\left.\mathbf{g}\left(x_{i_{0}} m\right)\right)$ is not admissible and is not an element of $P_{N}$. However, we are assuming that the symbol $\left(I \backslash\left\{i_{0}, i_{1}\right\}, \mathbf{g}\left(x_{i_{0}} m\right)\right)$ is admissible, so that $i_{1}$ is the element preventing $\left(I \backslash\left\{i_{0}\right\}, \mathbf{g}\left(x_{i_{0}} m\right)\right)$ from being admissible and $\max \left(\mathbf{g}\left(x_{i_{0}} m\right)\right) \leq i_{1}$. Lemma 1.2 of [15] therefore guarantees the monomial equality $\mathbf{g}\left(x_{i_{0}} \mathbf{g}\left(x_{i_{1}} m\right)\right)=\mathbf{g}\left(x_{i_{1}} m\right)$ so that $\left(I \backslash\left\{i_{0}\right\}, \mathbf{g}\left(x_{i_{0}} m\right)\right)=\left(I \backslash\left\{i_{0}\right\}, \mathbf{g}\left(x_{i_{0}} x_{i_{1}} m\right)\right)$ and the symbols $\left(I \backslash\left\{i_{1}\right\}, m\right)$ and $(I \backslash$ $\left.\left\{i_{1}\right\}, \mathbf{g}\left(x_{i_{1}} m\right)\right)$ are each contained in the interval. Since $n=\mathbf{g}\left(x_{i_{0}} m\right)$, the symbol $\left(I \backslash\left\{i_{0}\right\}, m\right)$ is not comparable to $\left(I \backslash\left\{i_{0}\right\}, \mathbf{g}\left(x_{i_{0}} m\right)\right)$.

If $n=\mathbf{g}\left(x_{i_{1}} m\right)$ and $\max \left(I \backslash\left\{i_{1}\right\}\right) \geq \max \left(\mathbf{g}\left(x_{i_{1}} m\right)\right)$ then the symbol $\left(I \backslash\left\{i_{1}\right\}\right.$, $\left.\mathbf{g}\left(x_{i_{1}} m\right)\right)$ is not admissible and is not an element of $P_{N}$. However, we are assuming the admissibility of the symbol $\left(I \backslash\left\{i_{0}, i_{1}\right\}, \mathbf{g}\left(x_{i_{1}} m\right)\right)$ and it follows that the element $i_{0}$ is preventing the admissibility of $\left(I \backslash\left\{i_{1}\right\}, \mathbf{g}\left(x_{i_{1}} m\right)\right)$. We therefore have $\max \left(\mathbf{g}\left(x_{i_{1}} m\right)\right) \leq$ $i_{0}<i_{1}$ and via Lemma 1.2 of [15], the monomial equality $\left.\mathbf{g}\left(x_{i_{1}} m\right)\right)=\mathbf{g}(m)=m$. However, $\max (m) \leq i_{0}<i_{1}$ is a contradiction to the admissibility of the symbol $(I, m)$. Hence, $\left(I \backslash\left\{i_{1}\right\}, \mathbf{g}\left(x_{i_{1}} m\right)\right)$ must be admissible and contained in the open interior of the interval along with the admissible symbol $\left(I \backslash\left\{i_{0}\right\}, m\right)$. The symbol $\left(I \backslash\left\{i_{1}\right\}, m\right)$ is not comparable to $\left(I \backslash\left\{i_{1}\right\}, \mathbf{g}\left(x_{i_{1}} m\right)\right)$ due to the absence of the value $i_{1}$. The symbol $\left(I \backslash\left\{i_{0}\right\}, \mathbf{g}\left(x_{i_{0}} m\right)\right)$ is also not comparable to $\left(I \backslash\left\{i_{1}\right\}, \mathbf{g}\left(x_{i_{1}} m\right)\right)$, for if it were then either $\mathbf{g}\left(x_{i_{1}} m\right)=\mathbf{g}\left(x_{\varnothing} \mathbf{g}\left(x_{i_{0}} m\right)\right)=\mathbf{g}\left(x_{i_{0}} m\right)$ or $\mathbf{g}\left(x_{i_{0}} x_{i_{1}} m\right)=n=$ $\mathbf{g}\left(x_{i_{1}} m\right)$. The first equality is impossible since Lemma 3.7 guarantees that $\left\{i_{1}\right\}$ is the unique set containing one element for which $n=\mathbf{g}\left(x_{i_{1}} m\right)$. The second equality also can not occur since Lemma 1.2 of [15] guarantees the monomial equality $\mathbf{g}\left(x_{i_{0}} x_{i_{1}} m\right)=\mathbf{g}\left(x_{i_{1}} m\right)$ if and only if $\max (n) \leq \min \left(x_{i_{0}}\right)=i_{0}$, which would contradict the assumption that $\left(I \backslash\left\{i_{1}\right\}, n\right)$ is an admissible symbol.

If $n=\mathbf{g}\left(x_{i_{0}} x_{i_{1}} m\right) \neq \mathbf{g}\left(x_{i_{0}} m\right)$ then the symbols $\left(I \backslash\left\{i_{0}\right\}, \mathbf{g}\left(x_{i_{0}} m\right)\right)$ and $(I \backslash$ $\left.\left\{i_{1}\right\}, \mathbf{g}\left(x_{i_{1}} m\right)\right)$ are admissible and are contained in the open interior of the interval. Clearly, the symbols $\left(I \backslash\left\{i_{0}\right\}, m\right)$ and $\left(I \backslash\left\{i_{1}\right\}, m\right)$ are not comparable to $\left(I \backslash\left\{i_{0}, i_{1}\right\}\right.$, $\left.\mathbf{g}\left(x_{i_{0}} x_{i_{1}} m\right)\right)$ in this instance. 
For each of these four choices of $n$, the interval $[(J, n),(I, m)]$ has four elements.

Subcase 2.2: We now consider the two remaining orderings $i_{0}<i_{2} \leq i_{1}<i_{3}$ and $i_{2} \leq i_{0}<i_{1}<i_{3}$. Under each of these orderings, we have $\operatorname{deg}_{x_{i_{3}}}(m)=1$ and in light of Lemma 1.3 of [15] if $n \neq m$ we have $\max (n)<\max (m)=i_{3}$ and in turn that $\max (n) \leq i_{1}$.

If $n=m$, then the poset elements which are contained in the open interior of the interval are forced to be $\left(I \backslash\left\{i_{0}\right\}, m\right)$ and $\left(I \backslash\left\{i_{1}\right\}, m\right)$.

If $n=\mathbf{g}\left(x_{i_{0}} m\right)$ then $\max (n) \leq i_{1}$ implies that the symbol $\left(I \backslash\left\{i_{0}\right\}, \mathbf{g}\left(x_{i_{0}} m\right)\right)$ is not admissible and is not an element of $P_{N}$. However, we are assuming that the symbol $\left(I \backslash\left\{i_{0}, i_{1}\right\}, \mathbf{g}\left(x_{i_{0}} m\right)\right)$ is admissible, so that $\max \left(\mathbf{g}\left(x_{i_{0}} m\right)\right) \leq i_{1}$ and again using Lemma 1.2 of [15], we have $\mathbf{g}\left(x_{i_{0}} \mathbf{g}\left(x_{i_{1}} m\right)\right)=\mathbf{g}\left(x_{i_{0}} m\right)$. Therefore, $\left(I \backslash\left\{i_{0}\right\}, \mathbf{g}\left(x_{i_{0}} m\right)\right)=\left(I \backslash\left\{i_{0}\right\}, \mathbf{g}\left(x_{i_{0}} x_{i_{1}} m\right)\right)$ and the symbols $\left(I \backslash\left\{i_{1}\right\}, m\right)$ and $(I \backslash$ $\left.\left\{i_{1}\right\}, \mathbf{g}\left(x_{i_{1}} m\right)\right)$ are each contained in the interval. Since $n=\mathbf{g}\left(x_{i_{0}} m\right)$, the symbol $\left(I \backslash\left\{i_{0}\right\}, m\right)$ is not comparable to $\left(I \backslash\left\{i_{0}\right\}, \mathbf{g}\left(x_{i_{0}} m\right)\right)$.

If $n=\mathbf{g}\left(x_{i_{1}} m\right)$ then the symbol $\left(I \backslash\left\{i_{0}\right\}, m\right)$ is certainly contained in the closed interval. Further, $\left(I \backslash\left\{i_{1}\right\}, \mathbf{g}\left(x_{i_{1}} m\right)\right)$ must be admissible for were it not, then the assumption of admissibility for $\left(I \backslash\left\{i_{0}, i_{1}\right\}, \mathbf{g}\left(x_{i_{1}} m\right)\right)$ implies that

$$
i_{0} \geq \max \left(\mathbf{g}\left(x_{i_{1}} m\right)\right) \geq \min \left(\left(\mathbf{g}\left(x_{i_{1}} m\right)\right)\right) \geq i_{1},
$$

a contradiction to the initial stipulation that $i_{0}<i_{1}$. The symbol $\left(I \backslash\left\{i_{1}\right\}, m\right)$ is incomparable to $\left(I \backslash\left\{i_{0}, i_{1}\right\}, \mathbf{g}\left(x_{i_{1}} m\right)\right)$ and were $\left(I \backslash\left\{i_{0}\right\}, \mathbf{g}\left(x_{i_{0}} m\right)\right)$ comparable to $(I \backslash$ $\left.\left\{i_{0}, i_{1}\right\}, \mathbf{g}\left(x_{i_{1}} m\right)\right)$, then either $\mathbf{g}\left(x_{i_{1} m}\right)=\mathbf{g}\left(x_{\varnothing} \mathbf{g}\left(x_{i_{0}} m\right)\right)=\mathbf{g}\left(x_{i_{0} m}\right)$ or $\mathbf{g}\left(x_{i_{0}} x_{i_{1}} m\right)=$ $n=\mathbf{g}\left(x_{i_{0}} m\right)$. The first equality contradicts Lemma 3.7 and the second may be used to arrive at a contradiction to the admissibility of $\left(I \backslash\left\{i_{1}\right\}, \mathbf{g}\left(x_{i_{1}} m\right)\right)$. These arguments are similar to those used in the case when $n=\mathbf{g}\left(x_{i_{0}} m\right)$ and $\max \left(I \backslash\left\{i_{0}\right\}\right)<$ $\max \left(\mathbf{g}\left(x_{i_{0}} m\right)\right)$.

Again, for each of these three choices of $n$, the interval has four elements.

We now analyze the vector spaces which are present in the sequence $\mathscr{D}\left(P_{N}\right)$ at the level of individual poset elements. In order to do so, we recall the following combinatorial results. As is standard, we write $\bar{P}=P \backslash\{\hat{0}, \hat{1}\}$.

Theorem 4.4 ([4, 8]). If a bounded poset $P$ is EL-shellable, then the lexicographic order of the maximal chains of $P$ is a shelling of $\Delta(P)$. Moreover, the corresponding order of the maximal chains of $\bar{P}$ is a shelling of $\Delta(\bar{P})$.

Theorem 4.5 ([8]). Suppose that $P$ is a poset for which $\hat{P}=P \cup\{\hat{0}, \hat{1}\}$ admits an EL-labeling. Then $P$ has the homotopy type of a wedge of spheres. Furthermore, for any fixed EL-labeling:

(i) $\widetilde{H}_{i}(\Delta(P), \mathbb{Z}) \cong \mathbb{Z}^{\# \text { falling chains of length } i+2}$,

(ii) bases for $i$-dimensional homology (and cohomology) are induced by the falling chains of length $i+2$. 
In the analysis that follows, we again examine the dual poset $P_{N}^{*}$ and focus our attention on the collection of closed intervals of the form $[(I, m), \hat{0}]$, to each of which we apply Theorem 4.5. Indeed, for each admissible symbol $(I, m) \in P_{N}^{*}$ where $|I|=$ $q$, the open interval $((I, m), \hat{0})$ is homeomorphic to a sphere of dimension $q-1$ since $P_{N}$ is a CW poset. Further, the EL-labeling of $[(I, m), \hat{0}]$ guarantees that the unique generator of $\widetilde{H}_{q-1}\left(\Delta_{I, m}, \mathbb{k}\right)$ is induced by a unique falling chain of length $q+1$. In the discussion that follows, we use the EL-shelling of Definition 3.4 to produce a canonical generator of $\widetilde{H}_{q-1}\left(\Delta_{I, m}, \mathbb{k}\right)$ as a linear combination in which each facet of $\Delta_{I, m}$ occurs with coefficient +1 or -1 .

To begin, consider a maximal chain $(I, m) \lessdot \sigma \lessdot \hat{0}$ which is of length $q+1$ and appears in the dual closed interval $[(I, m), \hat{0}]$ and write the label of this chain as

$$
\left(l_{1}^{\sigma}, \ldots, l_{q}^{\sigma}, 0\right) .
$$

We note that $I=\left\{\left|l_{1}^{\sigma}\right|, \ldots,\left|l_{q}^{\sigma}\right|\right\}$ and write

$$
\varepsilon_{\sigma}=\operatorname{sgn}\left(\rho_{\sigma}\right) \cdot \operatorname{sgn}\left(\prod_{t=1}^{q} l_{q}\right)
$$

where $\rho_{\sigma} \in \Sigma_{q}$ is the permutation arranging the sequence

$$
\left|l_{1}^{\sigma}\right|, \ldots,\left|l_{q}^{\sigma}\right|
$$

in increasing order. We endow the corresponding chain $\sigma$ in $((I, m), \hat{0})$ with this sign $\varepsilon_{\sigma}$ and refer to it as the sign of $\sigma$.

The unique maximal chain $\tau$ in $[(I, m), \hat{0}]$ which has a decreasing label is the chain consisting of admissible symbols having at each stage a different monomial as their second coordinate and the sequence of sets

$$
I, I \backslash\left\{i_{q}\right\}, I \backslash\left\{i_{q-1}, i_{q}\right\}, \ldots,\left\{i_{1}, i_{2}\right\},\left\{i_{1}\right\}, \varnothing
$$

as their first coordinate. The unique falling chain $\tau \in[(I, m), \hat{0}]$ is therefore

$$
(I, m) \lessdot\left(I_{q}, m_{q}\right) \lessdot\left(I_{q-1, q}, m_{q-1, q}\right) \lessdot \cdots \lessdot\left(I_{2, \ldots, q}, m_{2, \ldots, q}\right) \lessdot\left(\varnothing, m_{1, \ldots, q}\right) \lessdot \hat{0}
$$

where $I=\left\{i_{1}, \ldots, i_{q}\right\}$ with $i_{1}<\ldots<i_{q}$ and for $j=1, \ldots, q$, the set $I_{j, \ldots, q}=$ $I \backslash\left\{i_{j}, \ldots, i_{q}\right\}$ and the monomial $m_{j, \ldots, q}=\mathbf{g}\left(x_{i_{j}} \cdots x_{i_{q}} m\right)$. The label of the chain $\tau$ is therefore

$$
\left(i_{q}, \ldots, i_{1}, 0\right)
$$

and is decreasing. If there were another such chain with decreasing label, then such a chain would be counted by Theorem 4.5 and $(\hat{0},(I, m))$ would not have the homotopy type of a sphere, a contradiction to the fact that $P_{N}$ is a CW poset. In the context of 
the shelling order produced by the EL-shelling above, the chain $\tau$ appears lexicographically last among all maximal chains in the dual interval and is therefore the unique homology facet of $\Delta_{I, m}$.

Definition 4.6. For an admissible symbol $(I, m)$, set

$$
f(I, m)=\sum_{\sigma \in((I, m), \hat{0})} \varepsilon_{\sigma} \cdot \sigma
$$

the linear combination of all maximal chains of the open interval $((I, m), \hat{0})$ with coefficients given by (4.2).

Viewing the maximal chains of $((I, m), \hat{0})$ as facets in the order complex $\Delta_{I, m}$ we now establish the following.

Lemma 4.7. The sum $f(I, m)$ is a $(q-1)$-dimensional cycle in $\widetilde{H}_{q-1}\left(\Delta_{I, m}, \mathbb{k}\right)$ which is not the boundary of any $q$-dimensional face.

Proof. The maximal chains in the open interval $((I, m), \hat{0})$ are each of length $q-1$, so that no $q$-dimensional faces are present in $\Delta_{I, m}$. Thus, $f(I, m)$ cannot be the boundary of a $q$-dimensional face of $\Delta_{I, m}$.

We now show that $f(I, m)$ is a $(q-1)$-dimensional cycle. Suppose that $\sigma$ is a maximal chain in $((I, m), \hat{0})$ and let $(J, n)$ be an element of said chain. We exhibit a unique chain $\sigma^{\prime}$ which also appears in $f(I, m)$ and differs from $\sigma$ only at the element $(J, n)$.

Indeed, consider the chain $(I, m) \lessdot \sigma \lessdot \hat{0}$ along with its subchain $\left(J_{1}, n_{1}\right) \lessdot(J, n) \lessdot$ $\left(J_{2}, n_{2}\right)$. In the proof of Theorem 4.3, each closed interval of length two was shown to be of cardinality four, and therefore there exists a unique $\left(J^{\prime}, n^{\prime}\right) \in\left[\left(J_{1}, n_{1}\right),\left(J_{2}, n_{2}\right)\right]$ which is not equal to $(J, n)$. Defining $\sigma^{\prime}$ by removing $(J, n)$ and replacing it with $\left(J^{\prime}, n^{\prime}\right)$, we have constructed the desired chain.

We claim that for the chains $\sigma$ and $\sigma^{\prime}$, the associated signs $\varepsilon_{\sigma}$ and $\varepsilon_{\sigma^{\prime}}$ are opposite to one another.

If $\left(J_{2}, n_{2}\right)=\hat{0}$ then $\left(J_{1}, n_{1}\right)=\left(\{j\}, n_{1}\right)$ for some $j$. Thus, $(J, n)=(\varnothing, n)$ and $\left(J^{\prime}, n^{\prime}\right)=\left(\varnothing, n^{\prime}\right)$ so that the chains $\sigma$ and $\sigma^{\prime}$ have the same corresponding permutation $\rho$. Since either $n_{1}=n$ or $n_{1}=n^{\prime}$, without loss of generality we assume that $n_{1}=n$ so that $n^{\prime}=\mathbf{g}\left(x_{j} n\right)$. Therefore, the subchain $(\{j\}, n) \lessdot(\varnothing, n) \lessdot \hat{0}$ has $-j$ as its label, while $(\{j\}, n) \lessdot\left(\varnothing, n^{\prime}\right) \lessdot \hat{0}$ has $j$ as its label. This is the only difference in the labels $\lambda(\sigma)$ and $\lambda\left(\sigma^{\prime}\right)$ and $\varepsilon_{\sigma} \neq \varepsilon_{\sigma^{\prime}}$ is forced.

If $\left(J_{2}, n_{2}\right) \neq \hat{0}$ then for each case that appears in the classification of intervals of length two described in the proof of Theorem 4.3 , we can compute $\varepsilon_{\sigma} \neq \varepsilon_{\sigma^{\prime}}$.

When the differential $d$ in the reduced chain complex $\widetilde{e}_{\bullet}\left(\Delta_{I, m}\right)$ is applied to the sum $f(I, m)$, each term appears twice with opposite signs, so that $d(f(I, m))=0$ making $f(I, m)$ a $(q-1)$-dimensional cycle in $\widetilde{H}_{q-1}\left(\Delta_{I, m}, \mathbb{k}\right)$ as claimed. 


\section{Proof of Theorem 2.4}

With the choice for the bases of the vector spaces in $\mathscr{D}\left(P_{N}\right)$ established, we now turn to the proof that the poset $P_{N}$ supports the minimal free resolution of $R / N$. We first analyze the action of the differential of $\mathscr{D}\left(P_{N}\right)$ when it is applied to an arbitrary basis element $f(I, m)$.

Lemma 5.1. The map $\varphi_{q+1}^{(I, m),(J, n)}$ sends a basic cycle $f(I, m)$ to $(-1)^{p+\delta_{m, n}} \cdot f(J, n)$, where $I=\left\{i_{1}, \ldots, i_{q}\right\}$, the relationship $I \backslash J=\left\{i_{p}\right\}$ holds and

$$
\delta_{m, n}= \begin{cases}1 & \text { if } m \neq n \\ 0 & \text { otherwise }\end{cases}
$$

Proof. Write $d$ for the simplicial differential in the reduced chain complex $\widetilde{e}_{\bullet}\left(\Delta_{I, m}\right)$. The open interval $((I, m), \hat{0})$ may be realized as the union of half-closed intervals $[(J, n), \hat{0})$, so that the order complex of each half-closed interval is a cone with apex $(J, n)$. Applying the differential to the sum of all facets contained in the interval produces the boundary of the cone, which in this case is the order complex of $((J, n), \hat{0})$. Indeed, when $d$ is applied to the sum

$$
v=\sum_{\sigma \in[(J, n), \hat{0})} \varepsilon_{\sigma} \cdot \sigma,
$$

the faces in which the element $(J, n)$ remains appear twice and have opposite signs as described in the proof of Lemma 4.7. Thus, the only faces that remain in the expansion of $d(v)$ are of the form $\bar{\sigma}=\sigma \backslash\{(J, n)\}$.

Precisely,

$$
\begin{aligned}
\varphi_{q+1}^{(I, m),(J, n)}(f(I, m)) & =\left[d\left(\sum_{\sigma \in[(J, n), \hat{0})} \varepsilon_{\sigma} \cdot \sigma\right)\right] \\
= & {\left[\sum_{\sigma \in[(J, n), \hat{0})} \varepsilon_{\sigma} \cdot \bar{\sigma}\right] } \\
= & {\left[\sum_{\bar{\sigma} \in((J, n), \hat{0})} \varepsilon_{\sigma} \cdot \bar{\sigma}\right] . }
\end{aligned}
$$

The facet $\bar{\sigma}$ has an associated permutation $\rho_{\bar{\sigma}} \in \Sigma_{q-1}$, and using elementary properties of permutation signs, we have $\operatorname{sgn}\left(\rho_{\sigma}\right)=(-1)^{p+1} \cdot \operatorname{sgn}\left(\rho_{\bar{\sigma}}\right)$, where $I \backslash J=\left\{i_{p}\right\}$. Considering the definition of $\varepsilon_{\sigma}$, for each $(J, n)$ for which $(I, m) \lessdot(J, n) \in P_{N}^{*}$ we 
now have

$$
\begin{aligned}
\varepsilon_{\sigma} & =\operatorname{sgn}\left(\rho_{\sigma}\right) \cdot \operatorname{sgn}\left(\prod_{t=1}^{q} l_{q}\right) \\
& =(-1)^{p+1} \cdot \operatorname{sgn}\left(\rho_{\bar{\sigma}}\right) \cdot \operatorname{sgn}\left(\prod_{t=2}^{q} l_{q}\right) \cdot \operatorname{sgn}\left(l_{1}\right) \\
& =(-1)^{p+1} \cdot \operatorname{sgn}\left(l_{1}\right) \cdot \varepsilon_{\bar{\sigma}} \\
& =(-1)^{p+\delta_{m, n}} \cdot \varepsilon_{\bar{\sigma}}
\end{aligned}
$$

since $\operatorname{sgn}\left(l_{1}\right)=1$ if $n \neq m$ and $\operatorname{sgn}\left(l_{1}\right)=-1$ if $n=m$.

Therefore, (5.1) becomes

$$
\begin{aligned}
\varphi_{q+1}^{(I, m),(J, n)}(f(I, m)) & =\left[\sum_{\bar{\sigma} \in((J, n), \hat{0})} \varepsilon_{\sigma} \cdot \bar{\sigma}\right] \\
& =\left[\sum_{\bar{\sigma} \in((J, n), \hat{0})}(-1)^{p+\delta_{m, n}} \cdot \varepsilon_{\bar{\sigma}} \cdot \bar{\sigma}\right] \\
& =(-1)^{p+\delta_{m, n}} \cdot\left[\sum_{\bar{\sigma} \in((J, n), \hat{0})} \varepsilon_{\bar{\sigma}} \cdot \bar{\sigma}\right] \\
& =(-1)^{p+\delta_{m, n}} \cdot f(J, n)
\end{aligned}
$$

which proves the lemma.

As described in Section 2, the map $\varphi_{q+1}$ is defined componentwise on the onedimensional $\mathbb{k}$-vectorspace $\mathscr{D}_{q+1,(I, m)}$ for each poset element $(I, m)$. Using the conclusion of Lemma 5.1, we immediately have

$$
\varphi_{q+1} \mid D_{q+1,(I, m)}=\varphi_{q+1,(I, m)}(f(I, m))=\sum_{(J, n) \lessdot(I, m)}(-1)^{p+\delta_{m, n}} f(J, n)
$$

where $I=\left\{i_{1}, \ldots, i_{q}\right\}$ and $i_{1}<\cdots<i_{q}$ and $J=I \backslash\left\{i_{p}\right\}$.

Recall that the poset map $\eta: P_{N} \longrightarrow \mathbb{N}^{n}$ is defined as $(I, m) \mapsto \operatorname{mdeg}\left(x_{I} m\right)$, so that we can homogenize the sequence of vector spaces $\mathcal{D}\left(P_{N}\right)$ to produce

$$
\mathcal{F}(\eta): 0 \longrightarrow F_{d} \stackrel{\partial_{d}^{\mathscr{F}(\eta)}}{\longrightarrow} F_{d-1} \longrightarrow \cdots \longrightarrow F_{1} \stackrel{\partial_{1}^{\mathscr{F}(\eta)}}{\longrightarrow} F_{0}
$$


a sequence of multigraded modules. More precisely, for $q \geq 0$ and a poset element $(I, m) \neq \hat{0}$ where $I=\left\{i_{1}, \ldots, i_{q}\right\}$ and $i_{1}<\cdots<i_{q}$, the differential $\partial^{\mathcal{F}(\eta)}$ acts on a basis element $f(I, m)$ of the free module $F_{q+1}$ via the formula

$$
\begin{aligned}
\partial_{q+1}^{\mathcal{F}(\eta)}(f(I, m)) & =\sum_{\left(J, n^{\prime}\right) \lessdot(I, m)}(-1)^{p+\delta_{m, n^{\prime}}} x^{\eta(I, m)-\eta\left(J, n^{\prime}\right)} \cdot f\left(J, n^{\prime}\right) \\
& =\sum_{(J, m) \lessdot(I, m)}(-1)^{p} x_{i_{p}} \cdot f(J, m)-\sum_{(J, n) \lessdot(I, m)}(-1)^{p} \frac{x_{i_{p}} m}{\mathbf{g}\left(x_{i_{p}} m\right)} \cdot f(J, n)
\end{aligned}
$$

where $p$ takes the same value as in (5.2), so that $I \backslash\left\{i_{p}\right\}=J$.

It remains to show that $\mathscr{F}(\eta)$ is a minimal exact complex, and to do so we identify it as the Eliahou-Kervaire resolution.

Definition 5.2. The Eliahou-Kervaire minimal free resolution [15] of a stable ideal $N$ is

$$
\mathcal{E}: 0 \longrightarrow E_{d} \stackrel{\partial_{d}^{\mathcal{E}}}{\longrightarrow} E_{d-1} \longrightarrow \cdots \longrightarrow E_{1} \stackrel{\partial_{1}^{\mathcal{E}}}{\longrightarrow} E_{0}
$$

where $E_{0}=R$ is the free module of rank one with basis 1 and for $q \geq 0, E_{q+1}$ has as basis the admissible symbols

$$
\left\{e(I, m): I=\left\{i_{1}, \ldots, i_{q}\right\}, \max (I)<\max (m)\right\} .
$$

When applied to a basis element, the differential of $\&$ takes the form

$$
\begin{aligned}
\partial_{q+1}^{\mathcal{E}}(e(I, m))= & \sum_{p=1}^{q}(-1)^{p} x_{i_{p}} \cdot e\left(I \backslash\left\{i_{p}\right\}, m\right) \\
& -\sum_{p=1}^{q}(-1)^{p} \frac{x_{i_{p}} m}{\mathbf{g}\left(x_{i_{p}} m\right)} \cdot e\left(I \backslash\left\{i_{p}\right\}, \mathbf{g}\left(x_{i_{p}} m\right)\right)
\end{aligned}
$$

where we define $e\left(I \backslash\left\{i_{p}\right\}, \mathbf{g}\left(x_{p} m\right)\right)=0$ when $\max \left(I \backslash\left\{i_{p}\right\}\right) \geq \max \left(\mathbf{g}\left(x_{p} m\right)\right)$ (i.e. the symbol is inadmissible).

We now are in a position to prove the main result of this paper.

Proof of Theorem 2.4. The Eliahou-Kervaire symbols that are admissible index the multigraded free modules in the complexes $\mathscr{E}$ and $\mathcal{F}(\eta)$ and therefore the generators of these modules are in one to one correspondence with one another. Further, comparing Definition 5.2 and (5.3), $\partial^{\mathcal{E}}$ and $\partial^{\mathscr{F}(\eta)}$ have identical behavior on basis elements. The minimality and exactness of $\mathcal{E}$ implies the minimality and exactness of $\mathcal{F}(\eta)$ so that $\mathcal{F}(\eta)$ is a minimal poset resoution of $R / N$. 


\section{A Minimal Cellular Resolution of $R / N$}

The technique which follows is an example of a general approach which interprets cellular resolutions of monomial ideals through the theory of poset resolutions. This approach is described in [10], and is distinct from both the method of [1] concerning stable modules and the method of [19] which is specific to stable ideals. We begin by recalling a fundamental result due to Björner.

Proposition 6.1 ([5, Proposition 3.1]). A poset $P$ is a $C W$ poset if and only if it is isomorphic to the face poset of a regular $C W$ complex.

In the case of the poset of admissible symbols $P_{N}$, we interpret Björner's proof explicitly to produce the corresponding regular CW complex $X_{N}$. On the level of cells, $\hat{0} \in P_{N}$ corresponds to the empty cell and each admissible symbol $(I, m)$ of $P_{N}$ corresponds to a closed cell $X_{I, m}$ of dimension $|I|$ for which $P\left(X_{I, m}\right)=[\hat{0},(I, m)]$. Taking $X_{N}=\bigcup X_{I, m}$ we have an isomorphism of posets $P\left(X_{N}\right) \cong P_{N}$. The regular CW complex $X_{N}$ also comes equipped with a $\mathbb{Z}^{n}$ grading by realizing the map $\eta: P_{N} \longrightarrow \mathbb{N}^{n}$ of Theorem 2.4 as a map $\eta: X_{N} \longrightarrow \mathbb{N}^{n}$ where a cell $X_{I, m} \mapsto \eta(I, m)=\operatorname{mdeg}\left(x_{I} m\right)$.

Example 6.2. The stable ideal $N=\langle a, b, c\rangle^{2}=\left\langle a^{2}, a b, a c, b^{2}, b c, c^{2}\right\rangle$ has minimal resolution supported by $X_{N}$, the regular CW complex depicted below, which has six 0 -cells, eight 1-cells and three 2-cells. The face poset of this cell complex $P\left(X_{N}\right)$ is isomorphic to the poset of admissible symbols $P_{N}$ given in Example 3.5.

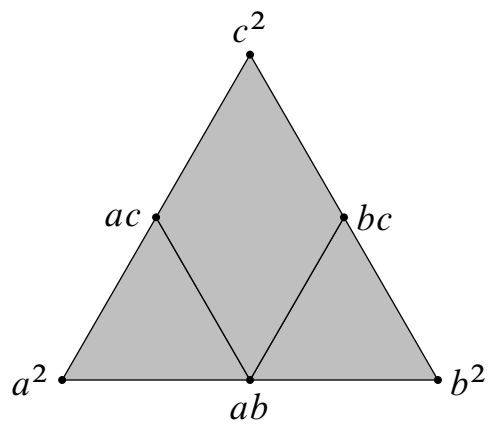

We recall the following well-known definition to which we incorporate the information given by the poset map $\eta$. For a more comprehensive view of cellular and CW resolutions, see $[1,3,24]$.

Definition 6.3. A complex of multigraded $R$-modules, $\mathscr{F}_{N}$, is said to be a cellular resolution of $R / N$ if there exists an $\mathbb{N}^{n}$-graded regular CW complex $X$ such that:

(i) For all $i \geq 0$, the free module $\left(\mathcal{F}_{N}\right)_{i}$ has as its basis the $i-1$ dimensional cells of $X$. 
(ii) For a basis element $e \in\left(\mathcal{F}_{N}\right)_{i}$, one has $\operatorname{mdeg}(e)=\eta(e)$,

(iii) The differential $\partial$ of $\mathscr{F}_{N}$ acts on a basis element $e \in\left(\mathcal{F}_{N}\right)_{i}$ as

$$
\partial(e)=\sum_{\substack{e^{\prime} \subset e \subset X \\ \operatorname{dim}(e)=\operatorname{dim}\left(e^{\prime}\right)+1}} c_{e, e^{\prime}} \cdot x^{\eta(e)-\eta\left(e^{\prime}\right)} \cdot e^{\prime}
$$

where $c_{e, e^{\prime}}$ is the coefficient of the cell $e^{\prime}$ in the differential of $e$ in the cellular chain complex of $X$.

With this definition in hand, we are now able to reinterpret Theorem 2.4 in our final result.

Theorem 6.4. Suppose that $N$ is a stable monomial ideal. Then the minimal free resolution $\mathscr{F}(\eta)$ is a minimal cellular resolution of $R / N$.

Proof. Conditions 1 and 2 of Definition 6.3 are clear from the structure of $X_{N}$, its correspondence to the poset $P_{N}$ and the construction of the resolution $\mathscr{F}(\eta)$. It therefore remains to verify that condition 3 is satisfied. The main result in [10] provides a canonical isomorphism between the complex $\mathscr{D}\left(P_{N}\right)$ and $\mathcal{C}\left(X_{N}\right)$, the cellular chain complex of $X_{N}$. Therefore, the differential of $\mathscr{F}(\eta)$ satisfies condition 3.

Acknowledgments. I am grateful to Amanda Beecher and Alexandre Tchernev for helpful questions and discussions during this project. Thanks to Jeff Mermin for careful reading of an early version of this document.

\section{Bibliography}

[1] Batzies, E., Welker, V., Discrete Morse theory for cellular resolutions. J Reine Angew Math. 2002;543:147-168.

[2] Bayer, D., Peeva, I., Sturmfels, B., Monomial resolutions. Math Res Lett. 1998;5(1-2): $31-46$.

[3] Bayer, D., Sturmfels, B., Cellular resolutions of monomial modules. J Reine Angew Math. 1998;502:123-140.

[4] Björner, A., Shellable and Cohen-Macaulay partially ordered sets. Trans Amer Math Soc. 1980;260(1):159-183.

[5] Björner, A., Posets, regular CW complexes and Bruhat order. European J Combin. 1984; 5(1):7-16.

[6] Björner, A., The homology and shellability of matroids and geometric lattices. Matroid applications. vol. 40 of Encyclopedia of mathematics and its applications. Cambridge: Cambridge University Press; 1992. p. 226-283. 
[7] Björner, A., Wachs, M., On lexicographically shellable posets. Trans Amer Math Soc. 1983;277(1):323-341.

[8] Björner, A., Wachs, M., Shellable nonpure complexes and posets. I. Trans Amer Math Soc. 1996;348(4):1299-1327.

[9] Clark, T., Poset resolutions and lattice-linear monomial ideals. J Algebra. 2010;323(4): 899-919.

[10] Clark, T., Tchernev, A., Regular CW complexes and poset resolutions. In preparation.

[11] Corso, A., Nagel, U., Specializations of Ferrers ideals. J Algebraic Combin. 2008;28(3): 425-437.

[12] Corso, A., Nagel, U., Monomial and toric ideals associated to Ferrers graphs. Trans Amer Math Soc. 2009;361(3):1371-1395.

[13] Eisenbud, D., Commutative algebra with a view toward algebraic geometry. vol. 150 of Graduate texts in mathematics. New York: Springer-Verlag; 1995.

[14] Eisenbud, D., The geometry of syzygies. A second course in commutative algebra and algebraic geometry. vol. 229 of Graduate texts in mathematics. New York: SpringerVerlag; 2005.

[15] Eliahou, S., Kervaire, M., Minimal resolutions of some monomial ideals. J Algebra. 1990;129(1):1-25.

[16] Grayson, D., Stillman, M., Macaulay2, a software system for research in algebraic geometry, Available from: http://www.math.uiuc.edu/Macaulay2/.

[17] Horwitz, N., Linear resolutions of quadratic monomial ideals. J Algebra. 2007;318(2): 981-1001.

[18] Lyubeznik, G., A new explicit finite free resolution of ideals generated by monomials in an R-sequence. J Pure Appl Algebra. 1988;51(1-2):193-195.

[19] Mermin, J., The Eliahou-Kervaire resolution is cellular. J Commut Algebra. 2010;2(1): $55-78$.

[20] Munkres, J., Elements of algebraic topology. Menlo Park, CA: Addison-Wesley Publishing Company; 1984.

[21] Nagel, U., Reiner, V., Betti numbers of monomial ideals and shifted skew shapes. Electron J Combin. 2009;16(2, Special volume in honor of Anders Björner):Research Paper 3 .

[22] Sinefakopoulos, A., On Borel fixed ideals generated in one degree. J Algebra. 2008; 319(7):2739-2760.

[23] Taylor, D., Ideals generated by monomials in an $R$-sequence [PhD thesis]. University of Chicago; 1966.

[24] Velasco, M., Minimal free resolutions that are not supported by a CW-complex. J Algebra. 2008;319(1):102-114. 


\section{Author Information}

Timothy B. P. Clark, Department of Mathematics and Statistics, Loyola University Maryland, Baltimore, MD, USA.

E-mail: tbclark@loyola.edu 